Contributed paper, presented at 1988 HIF Svmosium, Darmstadt, $\therefore$ Liermany, June $28-30,1988$

\title{
EXPERIMENTAL STUDIES OF ELECTRON BEAM TRANSPORT IN THE MARYLAND PERIODIC SOLENOID CHANNEL *
}
D. KEHNE, K. LOW, M. REISER, T. SHEA, C. R. CHANG, and Y. CHEN, ${ }^{* *}$
University of Maryland, College Park, MD 20742
CONF-8806101--8
DE89 005399

In the University of Maryland electron beam transport experiment, a $5 \mathrm{kV}, 0.2 \mathrm{~A}$ electron beam from a thermionic electron source (cathede radius $r_{c}=1.27 \mathrm{~cm}$ and cathode temperature $\mathrm{kT}=0.12 \mathrm{eV}$ ) is injected into a periodic focusing channel consisting of 38 solenoid lenses with period length $\mathrm{S}=13.6 \mathrm{~cm}$. The magnetic focusing fields of the channel, and hence the phase advance without space charge, can be varied over a wide range. We report emittance measurements and results of the effects of beam mismatch, misalignments, and nonlinear lens forces on beam transport and emittance growth.

\section{Introduction}

The thorough understanding of the transport of intense, highbrightness charged particle beams is essential to the develpment of heavy ion fusion drivers and other systems requiring such beams. Ongoing work at the University of Maryland Electron Beam Transport Experiment studies the periodic transport of a space charge dominated electron beam through a solenoid channel. Though an actual HIF system would use a quadrupole channel and heavy icns, the effects and instabilities discussed in this paper occur in both solenoid and quadrupole channels. The electron beam is used to provide an inexpensive and convenient model of an ion beam. Recent experiments hav $\epsilon$ emphasized the effects of beam mismatch, misalignments, lens nonlinearities, and the merging of multiple beamlets. The multiple beam work is reported elsewhere [1] and will not be included here.

* Research supporteu by the U.S. Department of Energy.

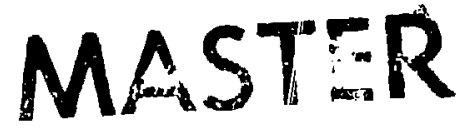

* Permanent address: Institute of Atomic Energy, Beijing, People's Republic of China. 
A schematic of the experiment is shown in Fig. 1. The electron source is a thermionic cathode (radius $r_{c}=1.27 \mathrm{~cm}$ ) in a Picrce type gun producing a $240 \mathrm{~mA}$ beam at an energy of $5 \mathrm{keV}$. The pulse length is $2 \mu \mathrm{sec}$ with a repetition rate of $60 \mathrm{~Hz}$. The first two solenoid lenses, $\mathrm{M1}$ and $\mathrm{M} 2$, are independently powered and are used to match the beam to the channel. The last 36 lenses, with period $\mathrm{S}=13.6 \mathrm{~cm}$, are connected in series and can be adjusted over a wide range of focusing strengths. The un-axis magnetic field of a single solenoid was carefully measured and fit to the analytic formula

$B(z)=B_{\circ} \frac{\exp \left(-0.5(z / b)^{2}\right)}{1+(z / a)^{2}}$

where $\mathrm{a}=4.4 \mathrm{~cm}$ and $\mathrm{b}=2.29 \mathrm{~cm}[2]$. Seven Rogowski coils placed at the gun and after lenses $\mathrm{C} 1, \mathrm{C} 9, \mathrm{C} 14, \mathrm{C} 22, \mathrm{C} 28$, and $\mathrm{C} 36$ monitor the beam current alonf the channel. In this way, beam transmission as a function of channel length can be determined. Details of emittance measurement (slit and pinhole combination) as well as other experimental details can be found in ref. [3].

\section{Beam Transport}

Successful transport of a space charge dominated beam depends heavily on the focusing strength of the channel lenses. When the focusing strength is low, the average radius of the beam increases. Thus, for focusing forces below a certain value, it is erpected that the beam radius will be so large that it will strike the beam tube, resulting in beam loss. On the other hand, when the focusing forces become too strong, mismatch and alignment errors as well as instabilities may again lead to beam loss, as explained below.

The quantitative value that will be used to define the channel focusing strength will be the phase advance per period when no space charge is present, denoted by $\sigma_{\circ}$ in units of degrees. In the presence of spacc charge, the phase advance per period is denoted by $\sigma$ and can be calculated using the smooth approximation formula $[4]$

$\sigma=\sigma_{\circ}\left(\sqrt{1+u^{2}}-u\right)$ 
where $\mathrm{u}=\mathrm{KS} / 2 \sigma_{\circ} \epsilon$. The generaiized perveance $\mathrm{K}$ is defined as $\left(\mathrm{I} / \mathrm{I}_{0}\right)\left(2 / \beta^{3} \gamma^{3}\right)$ where $\mathrm{I}_{0}=1.7 \times 10^{4} \mathrm{~A}$ for electrons, $\epsilon$ is the emittance, $\gamma$ is the relativistic mass factor, and $\beta$ is the beam velocity divided by the speed of light. Using the intrinsic cathode emittance of the beam, defined by $\epsilon_{i}=r_{c}\left(2 l \mathrm{~T} / \mathrm{eV}_{\circ}\right)^{1 / 2}=0.088 \mathrm{~mm}$-rad, where $\mathrm{kT}=0.12 \mathrm{eV}$ is the cathode temperature, and $V_{\circ}=5 \mathrm{kV}$ is the beam voltage, we obtain $\sigma=6.4^{\circ}$ for $\sigma_{\circ}$ $=70^{\circ}$.

In an effort to find the values of focusing strength that result in no beam loss along the length of the channel, the beam transmission was measured at yarious values of $\sigma_{\circ}$ between $40^{\circ}$ and $160^{\circ}$ corresponding to peak on-axis magnetic fields of 44 gauss and 157 gauss, respectively. At each value of $\sigma_{0}$, the matching lenses were adjusted to attain maximum beam transmission at the end of the channel. A plot of beam transmission versus $\sigma_{0}$ is shown in Fig. 2.

The window of $100 \%$ beam transmission extends over the range $55^{\circ} \leq$ $\sigma_{o} \leq 90^{\circ}$ where the depressed tune $\sigma / \sigma_{0}$ is approximately 0.1 . The beam loss below $55^{\circ}$ is attributable to the inability of the lenses to contain the beam within the radius of the beam pipe, as mentioned before. For the beam loss above $90^{\circ}$, three effects that play major roles are mismatch of the beam to the channel and the related envelope instability [5], misalignments of the gun and magnets, and nonlinearities present in the magnetic fields of the lenses. It should be understood that instabilities other than the envelope instability (e.g. third order instabilities [5]) may also cause some of the beam loss for $\sigma_{\circ} \geq 90^{\circ}$. Evidence of these instabilities simply can not be gleaned from the data that has thus far been coilected, though this by no means implies that they are not there.

First, consider beam mismatch as a function of $\sigma_{0}$. The sensitivity of beam radius to lens mismatch was studied by numerically solving the $K-V$ envelope equations for matching lens settings for both matched and mismatched beams at various values of $\sigma_{0}$. Assuming an ideal periodic channel, the matched beam and matching lens settings for a desired value of $\sigma_{0}$ were determined. Next, the relative magnetic field $\Delta B_{1} / B_{1}$ of the 
first matching lens was changed and the maximum radius of the resulting mismatched beam was determined. The mismatch is then cefined by the parameter

$$
\frac{\Delta R}{R}=\frac{\left(R_{\text {max }}\right)_{\text {mismatched }}-\left(R_{\text {max }}\right)_{\text {matched }}}{\left(R_{\text {max }}\right)_{\text {matched }}} .
$$

For a $1 \%$ error in the field of the first matching lens, i.e. $\Delta B_{1} / B_{1}=0.01$, one finds the radial mismatch $\Delta R / R$ as a function of $\sigma_{\mathfrak{o}}$ as shown in Fig. 3. The mismatch curve is relatively tlat below $\sigma_{0}$ values of $100^{\circ}$ but increases dramatically above $100^{\circ}$, peaking at $115^{\circ}$ and dropping off rapidly after that. The dramatic increase of the beam mismatch is related to the envelope instability. This matching lens sensitivity has in fact been noticed experimentally. Less than a $0.2 \%$ change in matching lens current will cause $20-40 \%$ additional beam loss for $\sigma_{\circ}$ between $110^{\circ}$ and $120^{\circ}$. Since accurate matching is difficult to achieve and maintain experimentally, this instability severely limits successful transport in this region.

Second, consider the presence of gun and lens misalignments in the channel. The effect of misalignments is to cause the beam centroid to wander away from the ideal system axis. Large misalignments can generally be avoided by careful alignment of the system. Errors present due to the limit of the accuracy of the alignment can not be eliminated and must be considered carefully. Since these random errors can not be measured, statistical study is necessary.

Space charge forces are zero at the beam center; therefore, the beam centroid feels a force due only to the external focusing fields. These forces cause the beam to wander from the axis as if it were a single particle. By considering many offsets, the RMS amplitude of the particle oscillation after a specified number of lenses can be predicted. Given the total number of periods $\mathrm{N}$, the period length $\mathrm{S}$, the zero current phase advance $\sigma_{\circ}$, and the RMS offset of the $\mathrm{N}$ misalignments $\Delta_{r m s}$, the RMS amplitude of the centroid trajectory is given by

$$
A_{\mathrm{rms}}=2 \Delta_{\mathrm{rms}} \sqrt{N} \sin \left(\sigma_{o} S / 2\right) .
$$


Unfortunately, this equation becomes inaccurate for values of $\sigma_{0}$ over $90^{\circ}$. In this region, numerical solution of the equations of motion using nonlinear expressions for the magnetic focusing fields gives a more accurate idea of the effects of random misalignments. In order to get a fairly statistical result, a single particle code was run 150 times for each value of $\sigma_{0}$, each run using different random lens misalignments. Only translational lens misalignment was included, not lens tilt, and the gun was assumed to be aligned. The RMS value of the 150 final amplitudes, and the RMS and maximum values of the 150 maximum channel offsets were then determined. Plots of the analytical and numerical results, and the experimental misalignments at the end of the channel are given in Fig. 4. The estimated experimental accuracy of the alignment method [6], $\Delta_{r m s}=0.20 \mathrm{~mm}$, was used in the analytical and numerical calculations. Because the specific misaligned state of the actual system is not statistical, a sharp variation of beam displacement as : function of $\sigma_{\circ}$ is expected in the experimental data. This is also present to a lesser degree in the numerical results since they are limited to a finite number of runs. Unfortunately, experimertal data at only a few points along the channel, though useful to find an idea of the magritude of the offset, reveals very little about the alignment state itself. It does show, however, that the system is not aligned as well as was hoped. The cause of the beam offset, whether it is a large misalignment of a lens or two, less accuracy in lens alignment than previously believed, a misalignment of the gun, or some other factor, is still uncertain.

Finally, lens nonlinearities, studied in depth in previous papers [2], increase the net focusing force with distance from the beam axis. 'This causes the outermost particles of the beam to form a halo around the beam core. This helo, having a much greater radius and transverse velocity than the main part of the beam, has a tendency to be lost quickly, especially in the presence of beam offset. Experimentally, the halo appears at the end of the channel for $95^{\circ} \leq \sigma_{\circ} \leq 115^{\circ}$, but disappears at higher values hizving been lost to the beam pipe early in the channel. Since the effects of the envelope 
instability and lens nonlinearities manifest themselves over the same range of $\sigma_{\circ}$, it is still unclear which is the dominant loss mechanism.

\section{Emittance Measurements}

Emittance measurements taken when the channel was in a state of poor alignment yielded an emittance growth (measured emittance at end of channel/intrinsic emittance) of 1.7 at $\sigma_{\circ}=70^{\circ}$ for a beam with current I $=160 \mathrm{~mA}$ and temperature $\mathrm{kT}=0.11 \mathrm{eV}[7,8]$. After the realignment of the channel $|6|$, the end of channel emittance was found to be $0.127 \mathrm{~mm}$-rad $\pm 0.006 \mathrm{~mm}-\mathrm{rad}$, corresponding to an emittance growth of 1.4 , at $\sigma_{0}=$ $70^{\circ}$ for a beam with $\mathrm{I}=240 \mathrm{~mA}$ and $\mathrm{kT}=0.12 \mathrm{eV}$. Considering the $33 \%$ increase in beam current (and a corresponding increase in beam radius of approximately $20 \%$ ), this is a dramatic improvement. The growth of 1.4 is easily attributabie to the remaining misalignments in the system. As seen in Fig. 4, the bearn offset at the end of the channel is approximately 3-4 mm fcr $\sigma_{\circ}=70^{\circ}$. Due to this off-centering as well as mismatch oscillations, the outer part of the beam experiences the nonlinear lens forces which results in emittance growth. Computer code simulation of this experiment done by $\mathrm{H}$. Rudd [9] resulted in an emittance growth of 1.1 when the beam was perfectly centered (no misalignments) and matched. When an initial beam offset of 2 mm was put in the simulation, the emittance growth was found to be 1.34 which is very close to the experimental result.

\section{Conclusion}

Realignment of the solenoid lenses in the Maryland transport channel has allowed a careful study of beam transport over a wide range of focusing strengths. There is $100 \%$ transport of a beam with a depressed tune of $\sigma / \sigma_{\circ} \sim 0.1$ uver the range $55^{\circ} \leq \sigma_{\circ} \leq 90^{\circ}$. Beam loss for $\sigma_{\circ} \geq 90^{\circ}$ has been connected to lens nonlinearities (causing formation of a halo) and the envelope instability (extreme mismatch sensitivity for $105^{\circ} \leq \sigma_{\circ} \leq 120^{\circ}$ ). The realignment has resulted in an improvement of emittance growth from 1.7 to 1.4. The growth of 1.4 is attributable to lens nonlinearities and 
mismatch and is in good agreement with the simulation result of 1.34 for an off-centered beam.

\section{References}

(1) M. Reiser, C.R. Chang, D. Kehne, K. Low, and T. Shea "Charge Homogenization and Emittance Growth in a Nonuniform Space-Charge Dominated Electron Beam", to be published.

(2) P. Loschialpo, Ph.D. Dissertation, Univ. of Md., 1984; P. Loschialpo, W. Namkung, M. Reiser, and J. D. Lawson, J. Appl. Phys. 푸, 10 (1985).

[3] E. Chojnacki, M.S. thesis, Univ. of Md., 1984; M. Reiser, E. Chojnacki, P. Loschialpo, W. Namkung, J. D. Lawson, C. Prior, and G. P. Warner, Proc. of the 1984 Lin. Accel. Conf., GSI-84-11 (Darmstadt, W. Germany, 1984), p. 309.

4) M. Reiser, Part. Accel. 8, 167 (1978).

(5) I. Hofmann, L. J. Laslett, L. Smith, and I. Haber, Part. Accel. 13, 145 (1983).

[6] M. Reiser, J. McAdoo, D. Kehne, K. Low, and T. Shea; Proceedings of the 1987 IEEE Particle Accelerator Conference, p. 1135-1137.

(7) J. McAdoo, E. Chojnacki, P. Loschialpo, K. Low, M. Reiser, and J. D. Lawson, IEEE Trans. Nucl. Sci. NS - 32, 2632 (1985).

[8] M. Reiser, J. McAdoo, D. Kehne, K. Low, and J.D. Lawson; HIF Symposium, May 27-29, 1986; published in AIP Conf. Proc. 152, p. 186-206, 1986(Edited by M. Reiser, T. Godlove, and R. Bangerter).

[9] H. Rudd, I. Haber, C. R. Chang, D. Kehne, K. Low, M. Reiser, and T. Shea, "Comparison of Simulaiion, Theory, and Measurement for the Maryland Transport Experiment ${ }^{n}$, elsewhere in this Proc.

\section{DISCLAIMER}

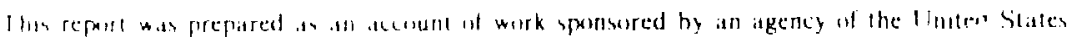

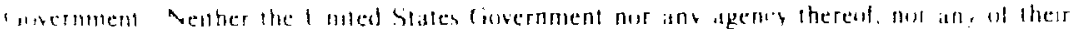

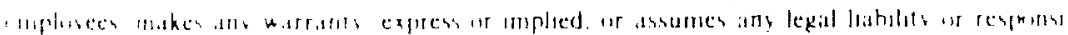

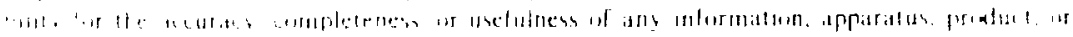

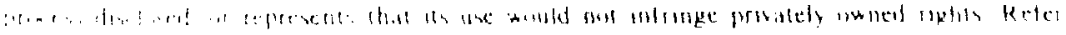

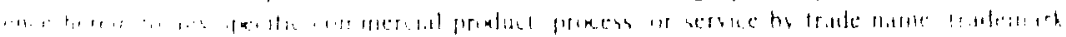

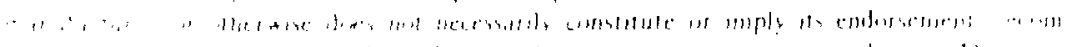

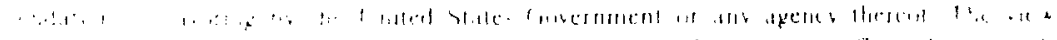

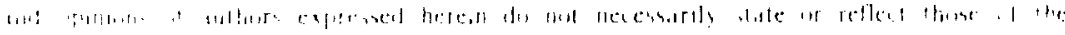

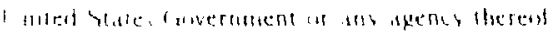




\section{FIGURE CAPTIONS}

Fig. 1. Schematic of the full experiment showing relevant viewports and diagnostics; current monitors are placed at the gun and after lenses $\mathrm{C} 1, \mathrm{C} 9, \mathrm{C} 14, \mathrm{C} 22, \mathrm{C} 28$, and $\mathrm{C} 36$.

Fig. 2. Fraction of beam transported after 9,22 , and 36 lenses as a function of phase advance $\sigma_{0}$.

Fig. 3. Beam mismatch caused by a $1 \%$ error in the current of matching lens $\mathrm{M} 1$ at different values of $\sigma_{\circ}$ ( $\mathrm{K}-\mathrm{V}$ numerical simulation).

Fig. 4. Results of 150 single particle code runs showing the effect of random lens misalignments $\left(\Delta_{r m s}=0.205 \mathrm{~mm}\right.$ ) on a single particle or beam centroid and comparison with analytic theory and experiment. 


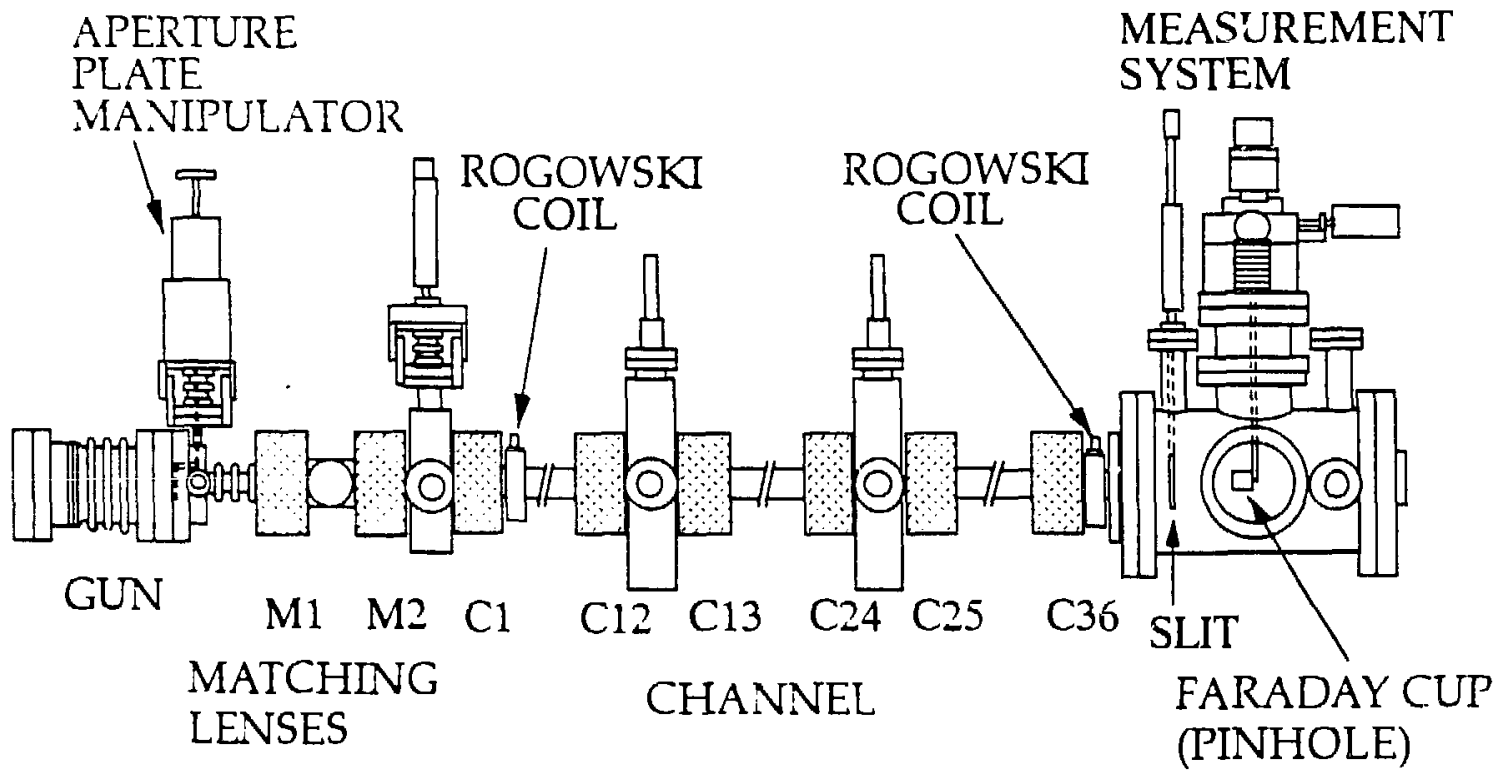




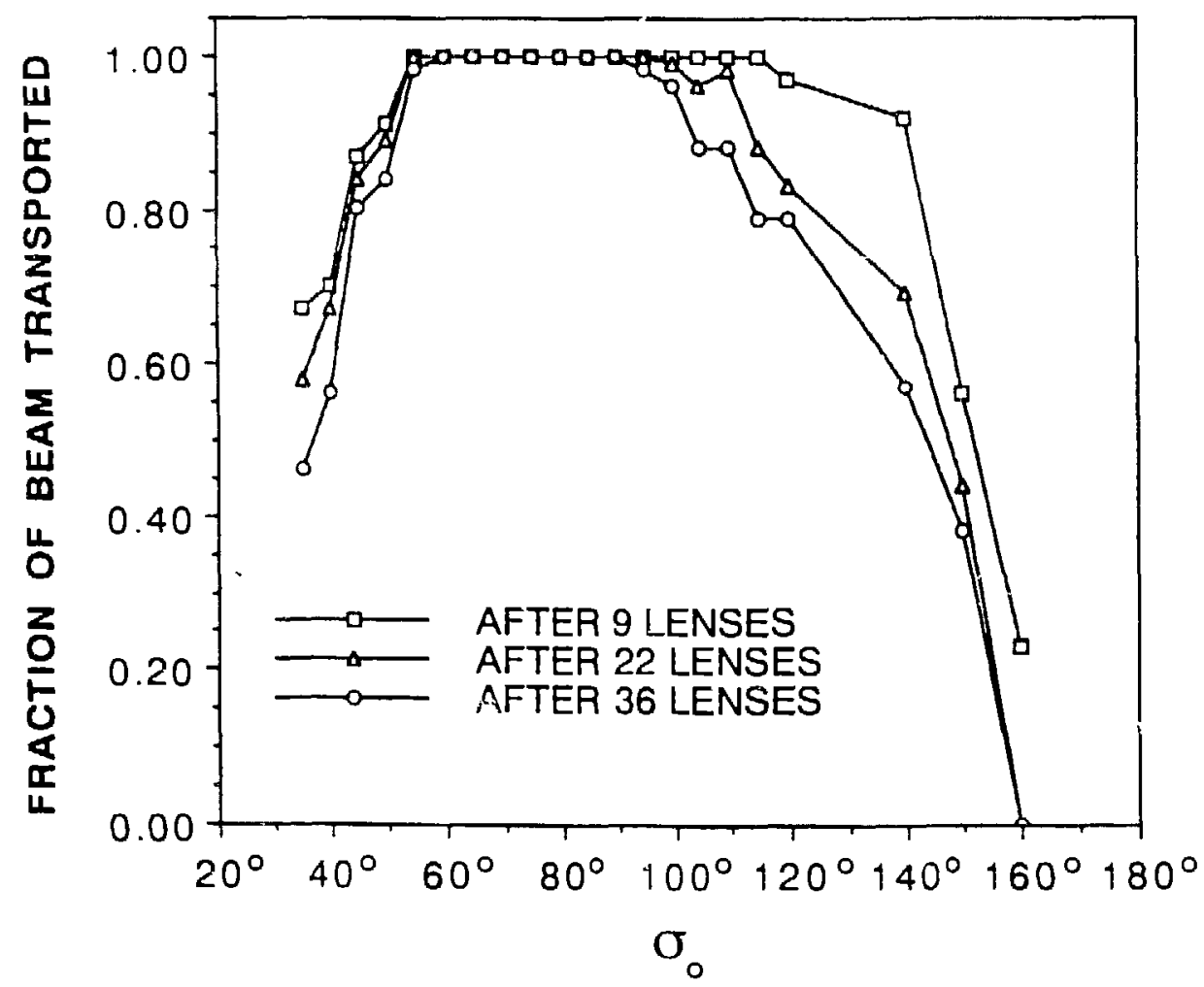




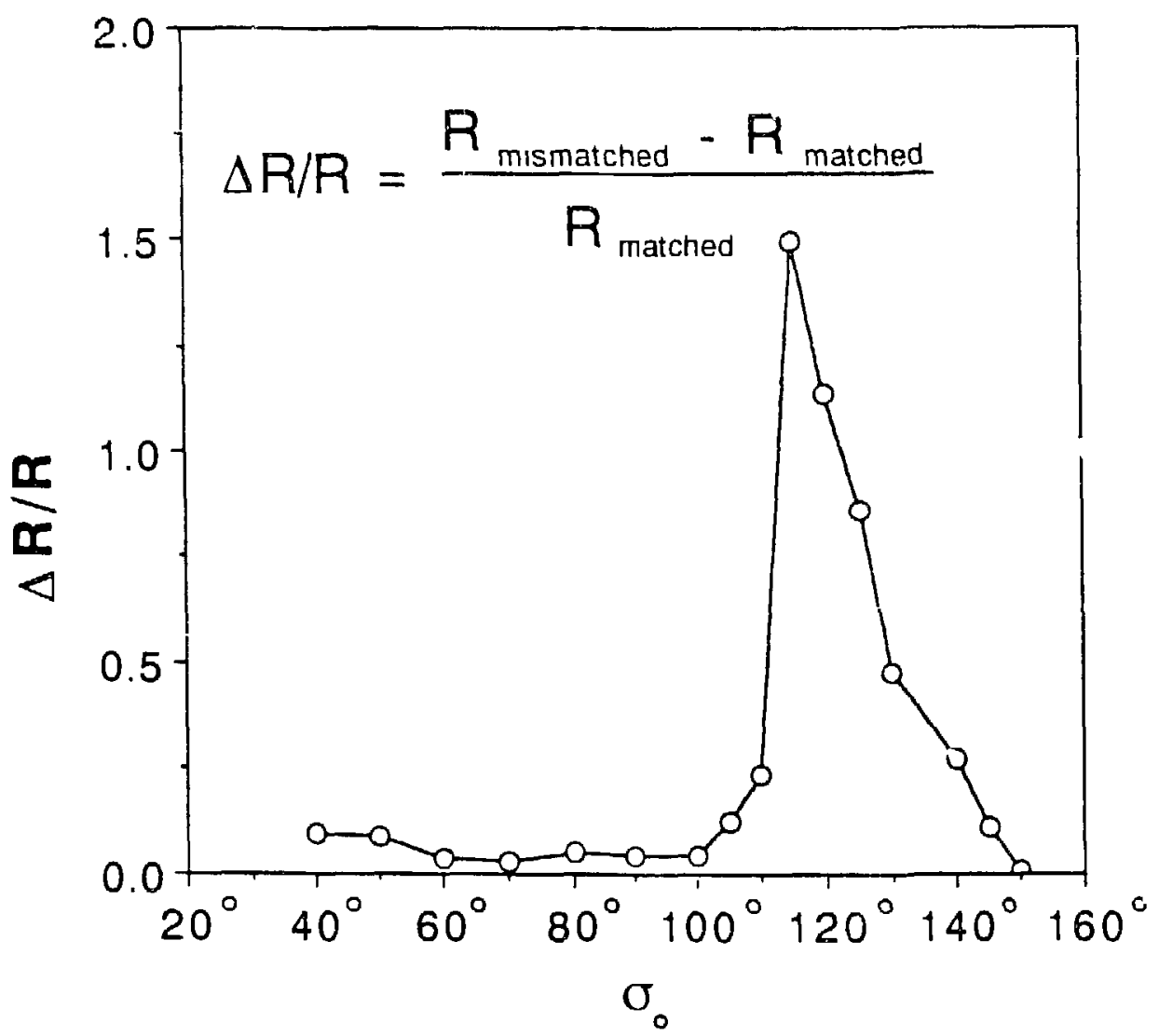




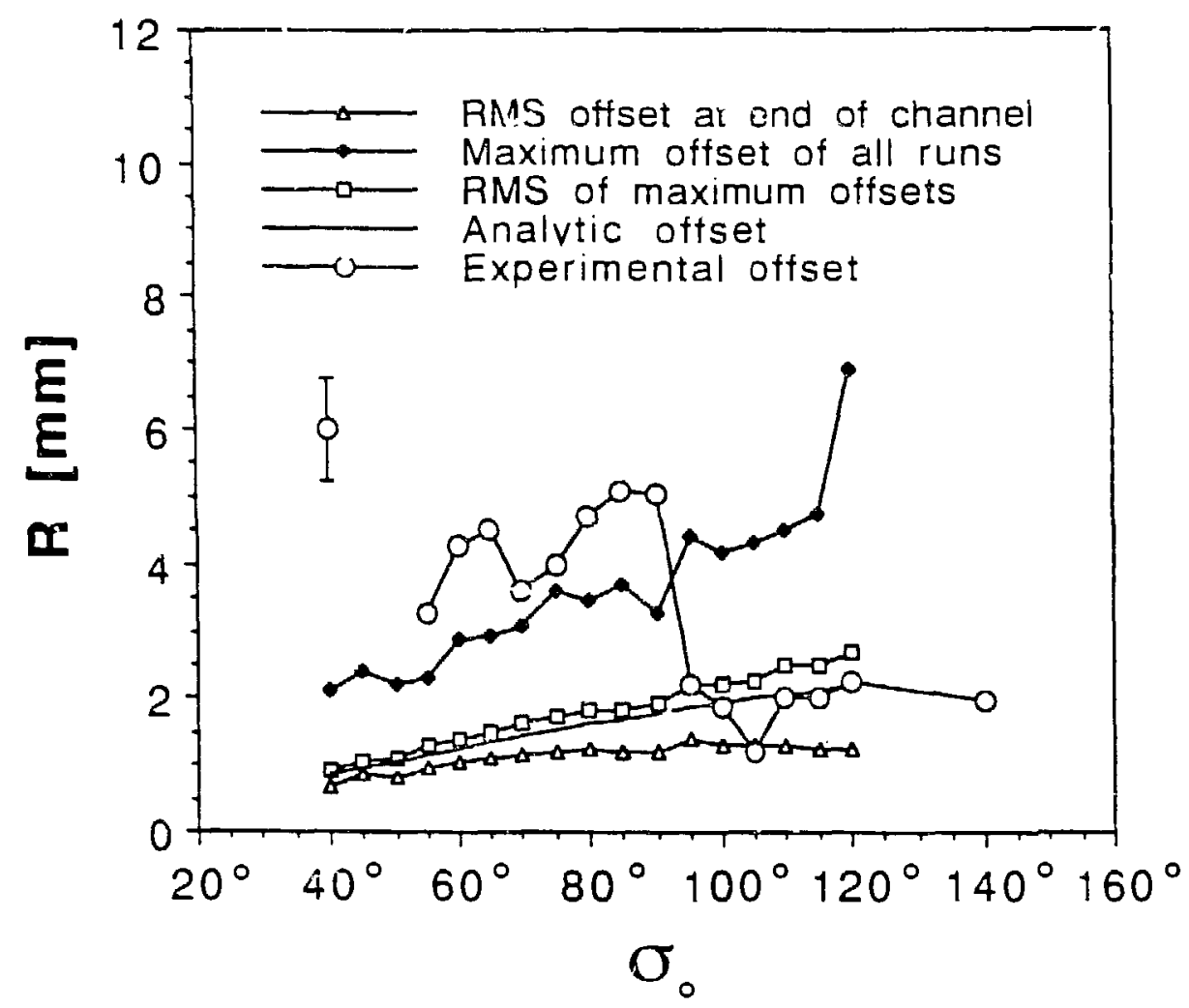

